\title{
La usabilidad en los portales webs de las pymes. Herramientas y usos para la comunicación empresarial
}

\author{
María GARCÍA GARCÍA \\ Universidad de Extremadura \\ mgargar@alcazaba.unex.es \\ María Victoria CARRILlo DURÁN \\ Universidad de Extremadura \\ mvcardur@alcazaba.unex.es \\ Ana CAstillo DíAz \\ Universidad de Extremadura \\ acasdia@alcazaba.unex.es
}

Recibido: $16 / 07 / 2012$

Aceptado: 22/10/2012

\section{Resumen}

La usabilidad se entiende como uno de los pilares básicos sobre los que se construyen las estrategias de comunicación empresarial online. La multiplicación de plataformas (redes sociales, blogs..), mediante las que una empresa puede comunicarse con los públicos ha tenido como consecuencia una menor atención de las organizaciones a las sedes webs corporativas. Las pequeñas y medianas empresas (PYMES) son empresas con un gran peso en el tejido empresarial de la mayoría de países europeos pero con grandes limitaciones presupuestarias. Esta investigación ha revisado las principales herramientas que utilizan las PYMES para disponer de sedes webs usables que fomenten una mejor comunicación con sus públicos de interés. Los resultados demuestran una atención escasa hacia la usabilidad.

Palabras clave: Usabilidad, PYMES, Internet, comunicación.

\section{The usability of web portals in the SMEs. Tools and uses for enterprise communication}

\begin{abstract}
The usability is understood as one of the pillars on which are built online business communication strategies. New communication platforms (social media, blogs...) are becoming more important in the online communication and, as consequence, organizations are paying less attention to their corporate websites. Small and medium enterprises (SMEs) are companies with a large weight in the economies of the most European countries but they are limited by budgets constraints. This research had reviewed the main tools used by SMEs to provide usable websites that encourage a better communication with their stakeholders. Results show limited attention to usability.

Key words: Usability, SMEs, Internet, communication.

\section{Referencia normalizada}

GARCÍA GARCÍA, María; CARRILLO DURÁN, M. Victoria y CASTILLO DÍAZ, Ana (2012): "La usabilidad en los portales webs de las pymes. Herramientas y usos para la comunicación empresarial". Estudios sobre el mensaje periodístico. Vol. 18, núm. especial octubre, págs.: 365-375. Madrid, Servicio de Publicaciones de la Universidad Complutense.
\end{abstract}

Sumario: 1. Introducción. 2. Las PYMES en Internet; 2.1. La usabilidad para las PYMES. 3. Metodología; 3.1. Análisis de contenidos; 3.2. Análisis factorial. 4. Discusión; 4.1. Discusión del análisis de contenidos; 4.2. Discusión del análisis factorial; 4.2.1. Factor 1. Navegabilidad; 4.2.2. Factor 2. Contenido. 5. Conclusiones. 6. Referencias bibliográficas. 


\section{Introducción}

Como punto de inicio es necesario reconocer la importancia de las pequeñas y medianas empresas (PYMES) dentro del mundo de las organizaciones. Son empresas con entidad propia, diferenciadas de las grandes compañías cuyas características intrínsecas les hacen demandar estrategias a su medida. Aunque cada día más estudios abordan las particularidades de las PYMES (Mitchell et al., 2010, entre otros) Internet ha abierto una nueva brecha entre grandes empresas y organizaciones de pequeño y mediano tamaño.

En el contexto online en el que se desarrollan las estrategias de comunicación actuales se puede decir que la usabilidad es una característica intrínseca al medio Internet (Da Silva y Alwi, 2005; Huertas Roig y Xifrá Triadú, 2009) y que, por tanto, sustentará las estrategias de comunicación empresarial en la World Wide Web. Sin embargo existe un gran desconocimiento sobre la predisposición de las empresas y organizaciones a disponer de sedes webs corporativas usables.

La preocupación por diseñar estrategias de comunicación que incluyan la usabilidad no es nueva. Estudios precedentes avalan la necesidad de integrar la usabilidad como un aspecto estratégico en el diseño (Sutcliffe, 2002) y la comunicación corporativa (Pollach, 2005) entre empresas y públicos.

En este estudio se pretende analizar la usabilidad de las sedes webs de las PYMES desde la perspectiva de la comunicación online y como forma de propiciar una experiencia positiva para el usuario. Para conseguir dicho objetivo se han seleccionado estadísticamente 231 sedes webs de PYMES ubicadas en la región española de Extremadura en las que se ha realizado un análisis de contenidos y un análisis factorial. Gracias a la verificación de las herramientas que utilizan las PYMES en sus sedes webs que más favorecen la usabilidad y a la comprobación de su agrupación, se puede ofrece una visión global de la situación actual que, a su vez, pueden servir de guía para la implantación de sedes webs usables en las PYMES.

\section{Las PYMES en Internet}

De acuerdo con la Comisión Europea (2006) las PYMES son aquellas empresas que emplean a menos de 250 trabajadores, tienen una facturación inferior a 50 millones de euros y/o un balance anual inferior a 43 millones de euros. Según el Directorio Central de Empresas (INE, 2012), a 1 de enero del año 2011 había en España 3.243.185 PYMES.

En el estudio realizado por SAGE (2011), las empresas españolas creen que la principal ventaja de estar presentes en la web es la posibilidad de acceder a sus negocios online y admiten que disponen de una página web corporativa que tiene una función principalmente informativa. El uso de las redes sociales en el ámbito profesional es todavía escaso, el 70,22\% declara tener perfil en la red social Facebook.

Tanto en España como en la comunidad extremeña, de donde procede la muestra tomada para este estudio, se observa una tendencia alcista en lo que a PYMES que disponen de sedes webs se refiere (INE, 2012). En Extremadura el 99,96\% de las empresas son PYMES, sumando así 65.463 en total (INE, 2012). 


\subsection{La usabilidad para las PYMES}

La usabilidad es un concepto que suele asociarse a características únicamente tecnológicas de la sede web. Así, puede ser definida como "estar seguro de que algo funciona bien: que una persona con habilidades medias pueda utilizar una cosa con un fin concreto sin terminar frustrado" (Krug, 2000). Sin embargo, el enfoque que adopta esta investigación es menos tecnológico y se centra en la aportación que la usabilidad puede hacer para favorecer la comunicación entre la organización y sus públicos con el fin de establecer relaciones duraderas. Huertas Roig y Xifra Triadú (2009: 259) resumen la aportación de la usabilidad a la comunicación en las organizaciones al decir que las "sedes de pequeñas dimensiones, iconografía muy básica y fácil uso, crean una buena imagen de marca. En cambio, sedes extensas y complicadas, de lenta apertura y que comportan confusión en la estructura interna, suponen la creación de actitudes no tan positivas o incluso desfavorables".

Los problemas de usabilidad pueden suponer que el usuario de por terminada la interacción con el sitio (Sutcliffe, 2002). Desde este punto de vista, puede decirse que el concepto de usabilidad está directamente relacionado con la satisfacción del usuario (Montero, 2006), que se sentirá a gusto y continuará la navegación si la web es cómoda y satisface sus deseos.

Llegados a este punto habría que plantearse qué entienden las PYMES por usabilidad. Según Rouke (2010) las PYMES entienden por usabilidad el hecho de utilizar herramientas gratuitas como Google Analytics para rastrear y monitorizar la actividad del visitante o probar versiones diferentes de la sede web corporativa mediante herramientas gratuitas como Google Website Optimiser.

\section{Metodología}

Este estudio pretende analizar la usabilidad de las sedes webs de las PYMES desde la perspectiva de la comunicación. Para ello se ha llevado a cabo una revisión de 231 sedes webs corporativas ubicadas en la región española de Extremadura. La muestra procede de la base de datos SABI que, en el mes de julio de 2011, incluía 465 PYMES ubicadas en la región y con sede web activa en el año 2009. De ellas, aplicando un error máximo de un $2 \%$ con un nivel de confianza del $95 \%$, se seleccionaron aleatoriamente 231.

Para conseguir este objetivo se ha realizado un análisis dividido en dos pasos.

\subsection{Análisis de contenidos}

En primer lugar, se han analizado los mensajes expuestos en las sedes webs corporativas estudiadas mediante un análisis de contenidos. Dicha técnica consiste en "la descripción objetiva, sistemática y cuantitativa del contenido manifiesto de la comunicación" (Berelson, 1952). Mediante el análisis de contenidos se han analizado fragmentos de texto (llamados indicadores) segmentados en unidades que posteriormente fueron codificados. Estos fragmentos de texto no son categorías independientes, sino que forman parte del modelo propuesto fruto de exhaustiva revisión bibliográfica (véase Anexo I y figura 1). 


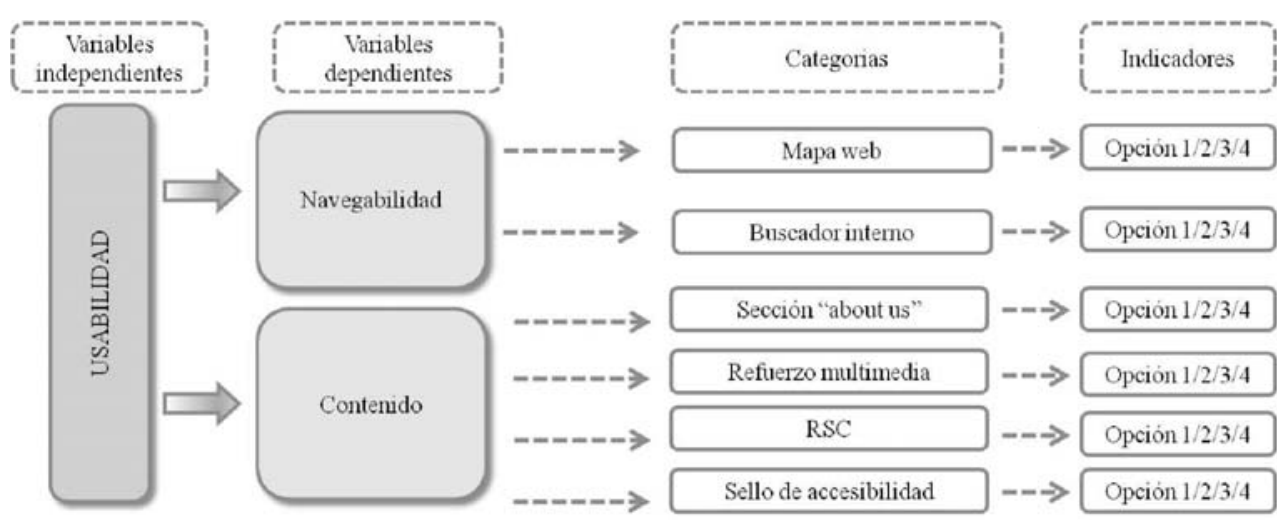

Figura 1: relación entre variables, categorías e indicadores. Fuente: elaboración propia

De acuerdo con la figura 1, el modelo se organiza en torno a diversas variables que se agrupan y dependen entre sí de forma más o menos directa. La gestión conjunta de todas las variables determinará la predisposición de la organización a disponer de una sede web usable. Así, los resultados obtenidos permiten conocer la situación de las PYMES extremeñas en relación con la variable usabilidad.

La variable independiente del estudio es, por tanto, la usabilidad. Esta variable independiente se configura mediante la suma positiva de las variables dependientes, categorías e indicadores que la conforman.

De la variable independiente (usabilidad) se desprenden las variables dependientes (que posteriormente serán denominados factores en el análisis factorial) que son la navegabilidad y el contenido.

Estos factores se materializan en las unidades de contexto (Andreú, 2000) o categorías que deberán ser examinadas para poder caracterizar y codificar los indicadores. Es decir, las variables y categorías aportarán la información necesaria del contexto para el análisis de los fragmentos de texto o indicadores.

Es importante hacer hincapié en la interrelación de los indicadores. Los indicadores se han adscrito a una categoría (Anexo I y figura 1) en función de lo expuesto en la literatura revisada, pero es importante no circunscribirlos exclusivamente a una categoría ni hacerlos excluyentes puesto que la suma positiva de todos estos indicadores dará lugar a una predisposición favorable hacia la usabilidad. La división ha sido necesaria para realizar el análisis de contenidos pero conviene destacar que se complementan y están interconectados, tal y como se comprobará posteriormente en el análisis factorial.

\subsection{Análisis factorial}

La revisión de la literatura vigente (Anexo I) valida el modelo y las categorías formuladas desde el punto de vista teórico. Sin embargo, es necesario comprobar si se ajustan o no a la realidad de las PYMES, saber si las PYMES asocian esos indicadores a las variables presentadas y si se relacionan entre ellos de la misma forma que en el modelo propuesto. Para ello se ha realizado un análisis factorial que se puede defi- 
nir como "un método multivariante que intenta explicar un conjunto de variables observables mediante un número reducido de variables no observables llamadas factores" (León Duarte, 2005: 56).

De esta forma, el análisis factorial de los resultados obtenidos en el análisis de contenidos ha permitido comprobar posibles modificaciones en el modelo teórico propuesto dando así el paso necesario de la teoría a la práctica.

Como su propio nombre indica, las variables dependientes propuestas han sido "factorizadas", por lo que dejarán de llamarse variables para ser denominadas factores. Estos nuevos factores pueden coincidir o no con las variables propuestas, por lo que será necesario renombrarlos en función de la nueva realidad que expliquen.

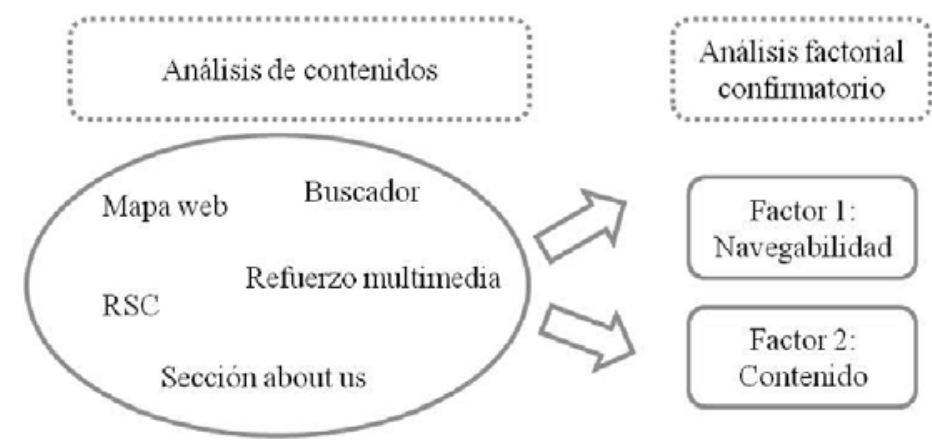

Figura 2: Paso del análisis de contenidos al análisis factorial. Fuente: elaboración propia

\section{Discusión}

La discusión de los resultados se va a realizar siguiendo los pasos dados para el análisis, de forma que primero se expondrán los resultados del análisis de contenidos y posteriormente se discutirán los resultados del análisis factorial. Ambas discusiones quedarán unidas en las conclusiones de la investigación.

\subsection{Discusión del análisis de contenidos}

La media alcanzada en cada indicador será un claro exponente de los resultados obtenidos en el análisis factorial (tabla 1). Partiendo del hecho de que todos los indicadores podían alcanzar una puntuación máxima de 4 puntos, las medias obtenidas permiten avanzar la escasa atención que prestan las PYMES a determinados aspectos de la usabilidad.

Tabla 1: medias obtenidas

\begin{tabular}{|l|c|}
\hline INDICADOR & MEDIA \\
\hline Mapa web & 1,31 \\
\hline Buscador interno & 1,31 \\
\hline Sección about us & 2,76 \\
\hline Refuerzo multimedia & 2,12 \\
\hline RSC & 1,32 \\
\hline
\end{tabular}

Fuente: elaboración propia 
En la variable dependiente navegabilidad, los dos indicadores propuestos (buscador y mapa web) han alcanzado una media cercana a la puntuación mínima. De acuerdo con Díaz Sánchez et al. (2008) estos dos indicadores son los principales mecanismos que facilitan la navegación en una sede web y mejoran la eficacia de la comunicación. Las puntuaciones conseguidas permiten atisbar resultados escasamente orientados a sedes webs navegables.

La estructura más observada en las webs analizadas ha consistido en una página principal desde la cual se accede a las secciones más importantes y cuyos niveles de profundidad no suelen exceder los dos o tres clics. La escasa presencia del buscador y del mapa web en las sedes webs estudiadas puede ser una consecuencia directa de la sencilla estructura alrededor de la cual articulan los contenidos las PYMES en sus sedes webs. Las PYMES entienden que todos los contenidos son fácilmente alcanzables y no ven la necesidad de ubicar y acompañar al usuario en el proceso de navegación.

Por su parte, la categoría contenido alberga al indicador con la media más alta de todas, el indicador "sección about us" con una media de 2,76 puntos sobre 4 posibles.

A pesar de esto puede decirse que las empresas que ofrecen a sus usuarios una sección about us, adolecen de un gran desconocimiento sobre cómo reflejar su cultura e identidad en la web. De acuerdo con Nielsen (2008) estas secciones están llenas de textos marketinianos, a los que el autor denomina "blah-blah" textos, en los que se pueden encontrar alusiones a la trayectoria de la empresa y referencias comerciales.

La combinación de texto y fotografía es la más empleada por las empresas analizadas, fotografías que unas veces acompañan al texto y otras simplemente forman parte del diseño de la interfaz. El recurso multimedia más utilizado es, por tanto, la fotografía presente en el $96,5 \%$ de las webs analizadas, mientras que solo un $2,3 \%$ de las webs analizadas ofrecían algún recurso en formato vídeo.

Sorprende sin embargo que la fotografía ocupe un lugar secundario en las sedes webs. Su función es eminentemente la de acompañar al texto. Este uso ornamental de la fotografía indica que la planificación del website ha sido concebida basándose en el texto, concediéndole una importancia secundaria a los elementos multimedia.

En relación a las políticas de RSC que llevan a cabo las PYMES, son todavía un misterio. Los públicos no pueden conocer las acciones de RSC que las empresas llevan a cabo sino las comunican. La media del indicador $(1,32)$ se sitúa próxima a la opción de no ofrecer ninguna información sobre las políticas de RSC que las PYMES realizan.

El hecho de no disponer de una sección específica en la que se comuniquen las acciones de RSC que lleva a cabo la empresa, o que no se nombre en ninguna sección no puede ser interpretado como una ausencia de interés de las PYMES en relación a las políticas de RSC ni obligatorias, exigibles o deseables (Silos y Galiano, 2011).

Las políticas de calidad y las acciones responsables con el medio ambiente son las que más comunican las PYMES estudiadas en sus sedes webs.

\subsection{Discusión del análisis factorial}

Una vez realizado el análisis de contenidos y con el fin de contrastar la teoría con la realidad práctica se realizó un análisis factorial confirmatorio. La pertinencia del aná- 
lisis factorial en la investigación quedó demostrada por diversas pruebas como son el determinante de la matriz de correlaciones, el test de esfericidad de Barlett, el índice KMO de Kaiser-Meyer-Olkin, la medida de adecuación de la muestra (MSA) y la matriz de correlaciones anti-imagen (García Jiménez, et al., 2000; Hair et al., 1999) que arrojaron resultados que permitían la realización del análisis.

Como ya se indicó anteriormente en la metodología estos nuevos factores necesitan ser renombrados en función de la realidad que expliquen. Los nuevos factores se compondrán de diversos indicadores que determinarán el nombre de la agrupación final.

Tan solo ha habido una modificación en los resultados respecto al modelo propuesto: la exclusión del indicador accesibilidad. Los resultados obtenidos en las diversas pruebas recomendaban la exclusión dicha variable para maximizar la pertinencia del análisis factorial.

\subsubsection{Factor 1. Navegabilidad}

Este factor queda configurado con las variables buscador interno y mapa web, que obtuvieron un autovalor de 1,36 y explican el $28,31 \%$ de la varianza.

La navegabilidad refleja el deseo de la empresa de que el usuario conozca la estructura de la web, se ubique fácilmente para poder llegar a todo el contenido y, si aún así no lo consigue, que pueda realizar búsquedas dentro de la página web. Es, por tanto, un exponente fundamental de la usabilidad.

\subsubsection{Factor 2. Contenido}

La coincidencia de las variables adscritas a este factor con las propuestas en el modelo teórico hace que se haya denominado al factor como contenido. Las variables asociadas al este segundo factor de la usabilidad son la sección about us, la RSC y el refuerzo multimedia. El conjunto de las variables tiene un valor propio de 1,24 y explican un $25,74 \%$ del total de la varianza.

Cómo se presenta la empresa, si comunica o no las acciones de RSC (tan en auge y tan importantes para la imagen de la empresa) y si el contenido está vinculado a los elementos multimedia son elementos clave a la hora de valorar el contenido de una sede web desde el punto de vista de la comunicación empresarial.

Las similitudes entre el modelo propuesto y los resultados obtenidos tras el análisis factorial refuerzan la validez de la investigación y la pertinencia de la metodología.

\section{Conclusiones}

Desde esta investigación se pretende conocer la importancia que le conceden las PYMES a la usabilidad en sus sedes webs, en el contexto de la comunicación empresarial. Los resultados obtenidos permiten afirmar que la usabilidad de las sedes webs corporativas de las PYMES estudiadas es muy básica.

La herencia de rutinas y formas de comunicación procedentes de los medios masivos y el desconocimiento de los requerimientos propios del medio Internet hace que las sedes webs corporativas descuiden aspectos tan importantes como es la ubicación del visitante durante el proceso de la navegación, mediante herramientas como el mapa 
web o el buscador. Este hecho ofrece una visión de las PYMES centradas en la exposición informativa en lugar del diálogo con el usuario.

El afán expositivo (escasamente interactivo) de las PYMES en sus sedes webs lleva a concluir que estas empresas diseñan portales webs con el fin de exhibir sus contenidos. No se aprecia una preocupación por diseñar portales web usables donde el usuario navegue por el portal sintiéndose asistido por la empresa.

Sin embargo cabe destacar que la sencillez del diseño de las sedes webs de las PYMES propicia una navegación cómoda. Obviamente este enfoque proviene de una óptica más tecnológica que comunicacional pero supone un aspecto positivo desde el que las PYMES podrían trabajar en el futuro para disponer de sedes webs más usables.

Las PYMES parecen cuidar de forma especial el contenido que muestran. A pesar de esto, los resultados obtenidos en este factor son claramente mejorables. La identidad corporativa se refleja en estos contenidos de forma superflua y manida.

No obstante podría decirse que las empresas son conscientes de la importancia que la identidad corporativa tiene en la web y la muestran mediante una sección específica. Las PYMES individualizan la sección y le otorgan un papel protagonista al que habría que añadir un trabajo de proyección de la identidad, mediante la información ofrecida, más acorde a la realidad y que refuerce la localidad y la cercanía de la empresa hacia sus públicos.

Estos contenidos se suelen acompañar de fotografías, como el principal recurso multimedia utilizado.

\section{Referencias bibliográficas}

ÁLVAREZ RODRÍGUEZ, María Luz et al. (2010): "Reputación y responsabilidad desde las webs corporativas" en Área abierta, $\mathrm{n}^{\circ} 26$. Disponible en: http://revistas.ucm.es/index.php/ARAB/article/view/ARAB1010230001A [consulta 09/05/2011]

BERELSON, Bernard (1952): Content Analysis in Comunication Research. Glencoe, Free Press.

CASTILLO DÍAZ, Ana (2007): La comunicación de las marcas universitarias españolas a través de sus sedes webs. Tesis doctoral, Universidad de Extremadura.

DA SILVA, R.V y SYED ALWI, S.F. (2006): “Online corporate brand image, satisfaction and loyalty". Brand management, 16 (3), 119-144

DÍAZ SÁNCHEZ, Estrella et al. (2008): "Evaluación de la eficacia de las páginas web: un análisis de contenido de las principales compañías aéreas", en Universidad, Sociedad y Mercados Globales / coord. por Emilio José de Castro Silva, José Díaz de Castro, pags. 279-293. Disponible en: http://dialnet.unirioja.es/servlet/articulo? codigo $=2751745$

DOU, Wenyu y KRISHNAMURTHY, Sandeep (2007): "Using Brand Web Sites to uild Brands Online: A Product vs. Service Brand Comparison". Journal of Advertising Research, 47(2), 193-206. Disponible en: http://fbstaff.cityu.edu.hk/mkwydou/Using\%20Brand $\% 20$ Websites $\% 20$ to $\% 20$ Build $\% 20$ Brands $\% 20$ Online_A $\%$ 
20Product\%20versus\%20Service\%20Brand\%20Comparison.pdf [consulta $13 / 06 / 2012]$

EUROPEAN COMMISION (2006): "The new SME definition". Disponible en: http://ec.europa.eu/enterprise/policies/sme/files/sme_definition/sme_user_guide_e n.pdf [consulta 20/01/2011]

GARCÍABORBOLLA, Amalia et al. (2005): "Empirical Evidence Concerning Smes' Corporate Websites: Explaining Factors, Strategies and Reporting", en The International Journal of Digital Accounting Research, vol. 5(10), 171-202.

GARCÍA JIMÉNEZ, Eduardo et al. (2000): Análisis factorial. Madrid, La Muralla.

GÓMEZ, Lina M. y CHALMETA, Ricardo (2011): "Corporate responsibility in U.S. corporate websites: A pilot study". Public Relations Review, $\mathrm{n}^{\circ}$ 37, 93-95.

HAIR, Joseph F. et al. (1999): Análisis multivariante. Madrid, Prentice Hall.

HASSAN MONTERO, Yusef y MARTÍN FERNÁNDEZ, Francisco Jesús (2003): "Guía para la evaluación heurística de sitios web". Nosolousabilidad.com. Disponible en: http://www.nosolousabilidad.com/articulos/heuristica.htm [consulta 17/05/2009]

HUERTAS ROIG, Asunción y Xifra Triadú, Jorge (2009): “¿Marcas o genéricos? La comunicación en línea de las marcas farmacéuticas". Zer. Revista de Estudios de Comunicación, vol. 14. Disponible en: http://www.ehu.es/zer/zer27/zer27-17-huertas.pdf [consulta 20/06/2011]

KRUG, Steve (2006): No me hagas pensar. Una aproximación a la usabilidad en la web. Madrid, Pearson Educación.

INSTITUTO NACIONAL DE ESTADÍSTICA(INE): www.ine.es

LEÓN DUARTE, Jaime A. (2005): Metodología para la detección de requerimientos subjetivos en el diseño de producto. Tesis doctoral, Universidad Politécnica de Cataluña. Disponible en: http://tdx.cat/handle/10803/6840 [consulta 06/06/2011]

MARTÍ PELLÓN, Daniel.; ÁLVAREZ RODRÍGUEZ, María Luz y DOMÍNGUEZ QUINTAS, Susana (2009), "Imagen corporativa web. Análisis del discurso de empresas de internet". Revista electrónica Razón y Palabra, no 69. Disponible en: http://www.razonypalabra.org.mx/comEstrategica/JUL30.html [consulta 03/07/2010]

MITCHELL, Richard; HUTCHINSON, Karise y BISHOP, Susan (2011): "Interpretation of the retail brand: an SME perspective". International Journal of Retail and Distribution Management, vol. 40(2).

NIELSEN, Jakob (2008): “About Us Information on Websites” Jakob Nielsen's Alertbox, 29 septiembre. Disponible en: http://www.useit.com/alertbox/about-uspages.html [consulta 20/07/2010].

POLLACH, I. (2005): "Corporate self-presentation on the WWW. Strategies for enhancing usability, credibility and utility". Corporate Communications: An international Review, 10(4), 285-301. 
ROUKE, Paul (2010): “What Does Usability Mean for SME's?" Disponible en: http:/www.paulrouke.co.uk/2010/02/04/what-does-usability-mean-for-smes/ [consulta 10/09/2012]tSAGE (2011): Radiografía de la Pyme 2011. Disponible en: http://blog.sage.es/wp-content/uploads/2011/03/Radiograf\%C3\%ADa-de-la-Pyme2011.pdf [consulta 21/07/2011]

SILOS, Jaime y GALIANO, Juan Pedro (2011): “Informe Forética 2011. Evolución de la responsabilidad social de las empresas en España". Disponible en: http://www.foretica.org/biblioteca/informes-foretica/doc_details/298-informe-foretica-2011-version-extendida-?lang=es [consulta 08/04/2011]

SUTCLIFFE, Alistair G. (2002): “Assessing the Reliability of Heuristic Evaluation for Website Attractiveness and Usability". Proceedings of the 35th Hawaii International Conference on System Sciences.

\section{ANEXO I. Ficha de análisis}

\begin{tabular}{|c|c|c|c|c|}
\hline ֻัญ & Categorías & Indicadores & 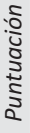 & Autores que han utilizado la categoría \\
\hline \multirow{8}{*}{ 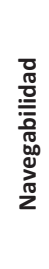 } & \multirow{4}{*}{$\begin{array}{l}\text { Dispone de } \\
\text { mapa web }\end{array}$} & Ambas aplicaciones siempre visibles & 4 & \multirow{4}{*}{$\begin{array}{l}\text { García Borbolla et al. (2005); Dou y } \\
\text { Krishnamurthy (2007); Díaz Sánchez et al. } \\
\text { (2008); Huertas Roig y Xifrá Triadú (2009) }\end{array}$} \\
\hline & & mapa web en la página principal & 3 & \\
\hline & & Indica ruta de navegación & 2 & \\
\hline & & No dispone & 1 & \\
\hline & \multirow{4}{*}{$\begin{array}{l}\text { Buscador } \\
\text { interno }\end{array}$} & Siempre visible & 4 & \multirow{4}{*}{$\begin{array}{l}\text { García Borbolla et al. (2005); Dou y } \\
\text { Krishnamurthy (2007); Díaz Sánchez et al. } \\
\text { (2008); Huertas Roig y Xifrá Triadú (2009) }\end{array}$} \\
\hline & & Accesible desde la home page & 3 & \\
\hline & & En alguna sección & 2 & \\
\hline & & No dispone & 1 & \\
\hline \multirow{16}{*}{ 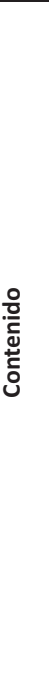 } & \multirow{4}{*}{$\begin{array}{l}\text { Dispone de } \\
\text { una sección } \\
\text { "about us }\end{array}$} & $\begin{array}{l}\text { Diversas facetas de la identidad (vision, } \\
\text { misión...) }\end{array}$ & 4 & \multirow{4}{*}{$\begin{array}{l}\text { Pollach (2005); Dou y Krishnamurthy } \\
\text { (2007) }\end{array}$} \\
\hline & & Presentación génerica de la empresa & 3 & \\
\hline & & $\begin{array}{l}\text { No dispone de sección about us pero hace } \\
\text { referencia a la identidad de la empresa en } \\
\text { la presentación o el inicio }\end{array}$ & 2 & \\
\hline & & No dispone & 1 & \\
\hline & \multirow{4}{*}{$\begin{array}{l}\text { Refuerzo } \\
\text { multimedia }\end{array}$} & Ambas herramientas & 4 & \multirow{4}{*}{$\begin{array}{l}\text { García Borbolla et al. (2005); Dou y } \\
\text { Krishnamurthy (2007); Castillo Díaz } \\
\text { (2007); Martí Pellón et al. (2009); Huertas } \\
\text { Roig y Xifrá Triadú (2009); Gómez y } \\
\text { Chalmeta (2011) }\end{array}$} \\
\hline & & Foto/audio/video corporación & 3 & \\
\hline & & Foto/audio/video productos & 2 & \\
\hline & & No dispone & 1 & \\
\hline & \multirow{4}{*}{ RSC } & $\begin{array}{l}\text { Sección propia accesible desde la home } \\
\text { page }\end{array}$ & 4 & \multirow{4}{*}{$\begin{array}{l}\text { Huertas y Xifrá (2009); Gómez y Chalmeta } \\
\text { (2011); Álvarez Rodríguez et al. (2010) }\end{array}$} \\
\hline & & Sección visible a partir de segundos niveles & 3 & \\
\hline & & Se nombra en alguna sección & 2 & \\
\hline & & No dispone & 1 & \\
\hline & \multirow{4}{*}{$\begin{array}{l}\text { Dispone de } \\
\text { sello } \\
\text { accesibilidad }\end{array}$} & Validada por la WAI & 4 & \multirow{4}{*}{$\begin{array}{l}\text { Montero y Martín Fernández (2003); Díaz } \\
\text { Sánchez et al. (2008) }\end{array}$} \\
\hline & & Dispone de versión móvil & 3 & \\
\hline & & Aplica algunas herramientas & 2 & \\
\hline & & No dispone & 1 & \\
\hline
\end{tabular}


Nota: este artículo es fruto de un Proyecto financiado gracias a la "Ayuda a consolidación de Grupos de Investigación”. 2012. Junta de Extremadura y Fondos Feder. Unión Europea.

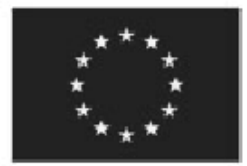

Proyecto financiado con

Fondos FEDER

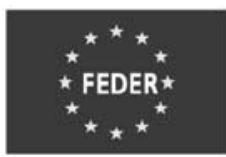

UNIÓN EUROPEA FONDO EUROPEO DE DESARROLLO REGIONAL: UNA MANERA DE HACER EUROPA
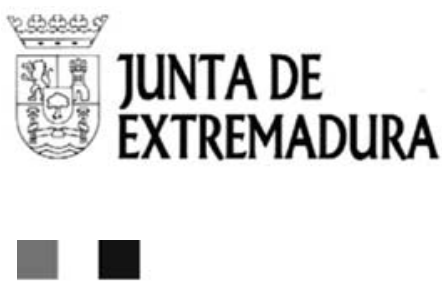

GOBIERNO DE EXTREMADURA

María GARCÍA GARCÍA

Universidad de Extremadura

mgargar@alcazaba.unex.es

\section{María Victoria CARRILLO DURÁN}

Universidad de Extremadura

mvcardur@alcazaba.unex.es

\section{Ana CASTILLO díAZ}

Universidad de Extremadura acasdia@alcazaba.unex.es 